Journal of The Korean Astronomical Society

43: $105 \sim 113,2010$ August

(C)2010 The Korean Astronomical Society. All Rights Reserved.

\title{
DYNAMICAL EVOLUTION OF THE M87 GLOBULAR CLUSTER SYSTEM
}

\author{
Sungsoo S. KIM ${ }^{1,2}$, Jihye Shin ${ }^{1}$, And Ho Jin ${ }^{2}$ \\ ${ }^{1}$ Department of Astronomy \& Space Science, Kyung Hee University, Yongin, Kyungki 446-701, Korea \\ ${ }^{2}$ School of Space Research, Kyung Hee University, Yongin, Kyungki 446-701, Korea \\ (Received April 20, 2010; Revised June 08, 2010; Accepted June 12, 2010)
}

\begin{abstract}
We study the dynamical evolution of the M87 globular cluster (GC) system using the most advanced and realistic Fokker-Planck (FP) model. By comparing our FP models with both mass function (MF) and radial distribution (RD) of the observed GC system, we find the best-fit initial (at M87's age of 2-3 Gyr) MF and RD for three GC groups: all GCs, blue GCs, and red GCs. We estimate the initial total mass in GCs to be $1.8_{-0.2}^{+0.3} \times 10^{10} \mathrm{M}_{\odot}$, which is about 100 times larger than that of the Milky Way GC system. We also find that the fraction of the total mass currently in GCs is $34 \%$. When blue and red GCs are fitted separately, blue GCs initially have a larger total mass and a shallower radial distribution than red GCs. If one assumes that most of the significant major merger events of M87 have ended by the age of 2-3 Gyr, our finding that blue (metal-poor) GCs initially had a shallower radial distribution supports the major merger scenario for the origin of metallicity bimodality.
\end{abstract}

Key words : stellar dynamics - Galaxy: globular clusters: general - Galaxy: evolution - Galaxy: formation - Galaxy: kinematics and dynamics

\section{INTRODUCTION}

Globular clusters (GCs) are the oldest stellar populations in galaxies. The mass function $\left(\mathrm{MF}^{*}\right)$ and radial distribution (RD) of the GC system in a galaxy evolves due to various internal and external processes such as stellar evolution, two-body relaxation, mass segregation, galactic tidal fields, and dynamical friction. Particularly, the initial GCMF is of interest since it can tell us about the mode of star formation and about the fraction of stars that formed inside GCs duringa the galaxy's formation.

The present-day globular cluster mass function of the Milky Way is indeed quite different from those of young cluster systems in other galaxies. The Galactic GC system presently has a log-normal MF with a peak at $M_{p}=2 \times 10^{5} \mathrm{M}_{\odot}$ and a dispersion of $\sigma\left(\log M / \mathrm{M}_{\odot}\right)=0.5$, while, for example, the MF of young cluster system in merging Antennae galaxies appears to follow a simple power-law function (Zhang \& Fall 1999). Shin, Kim, \& Takahashi (2008; SKT hereafter) calculated the dynamical evolution of hundreds of model GCs using a Fokker-Planck model, and found that the initial GCMF that best fits the observed GCMF of the Milky Way ${ }^{\dagger}$ is a lognormal function with a peak at $4 \times 10^{5} \mathrm{M}_{\odot}$ and a dispersion of 0.33 (a powerlaw function with a slope of 2.3 and a lower mass cut-

Corresponding Author: S. S. Kim

* By the MF of a GC system, we mean the number of clusters, not stars, as a function of mass.

†When fitting the observed GCMF, SKT considered the 'old halo' and 'bulge/disc' populations only. See their paper for details. off at $4 \times 10^{5} \mathrm{M}_{\odot}$ gave a similarly good fit as well). Their best-fit model has a total initial mass in GCs of $1.5 \times 10^{6} \mathrm{M}_{\odot}$, and $83 \%$ of this mass has evaporated during the lifetime of the Milky Way.

M87, a cD galaxy in the Virgo cluster, has the largest number of GCs among galaxies in the local universe. The total GC population in M87 is estimated to be about 15000 (Peng et al. 2008). M87 has a specific frequency (SN, the number of clusters per unit galaxy luminosity scaled for $M_{V}=15 \mathrm{mag}$ ) of 12.6 , while the Milky Way has $\sim 0.6$. M87's SN is $3-10$ times higher than those of other luminous early-type galaxies in the Virgo cluster (Peng et al. 2008), making M87 an outstanding outlier.

M87 has 3-10 times larger enclosed mass profile $M_{e n c}$ than the Milky Way at all galactocentric radii $R$ (see Fig. 1). When a GC is situated in a larger $M_{e n c}$, it evaporates more rapidly due to a smaller tidal radius $r_{t}\left(\propto R\left[M / M_{e n c}\right]^{1 / 3}\right)$ and migrates inward more quickly due a higher local field star density. Thus the GC system in M87 is expected to have undergone more significant dynamical evolution than that of the Milky Way.

In the present paper, we study the evolution of the M87 GC MF and RD using the same numerical method and procedure used by SKT for the Milky Way GC system. We perform Fokker-Planck (FP) calculations for 192 different initial conditions (mass, galactocentric radius, and orbit eccentricity; unlike in Shin et al., we do not consider an orbit inclination here because M87 does not have a disk). We then search a wide parameter space for the best-fit initial GC mass and radial 


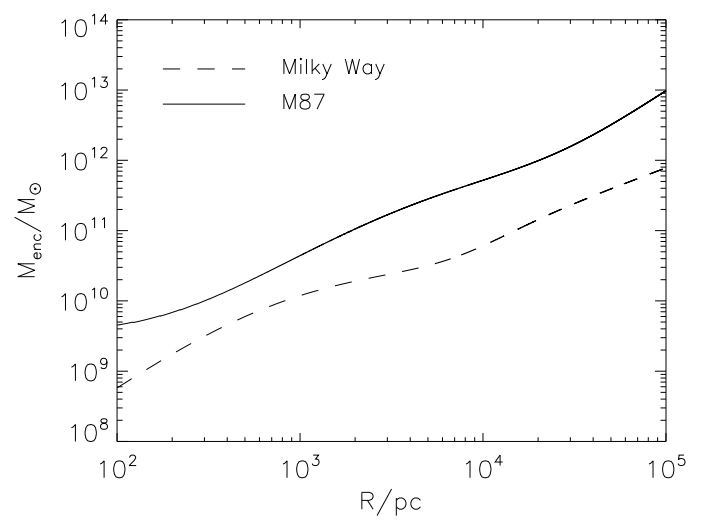

Fig. 1.- Enclosed masses (including both stellar and dark matter components) of the Milky Way (dashed) and M87 (solid) from Johnston, Spergel, \& Hernquist (1995) and McLaughlin (1999), respectively.

distributions that evolve into the observed, present-day Galactic GC distributions (MF and RD). We adopt a log-normal and a Schechter (Schechter 1976) function for the initial $\mathrm{MF}$, and a softened power-law function for the initial RD.

\section{THE M87 GLOBULAR CLUSTER CATA- LOG}

We will compare our FP calculations to observed M87 GC data presented in the ACS Virgo Cluster Survey (Côté 2004; Jordán et al. 2009), which is an imaging survey of 100 early-type galaxies in the Virgo cluster using the Advanced Camera for Surveys (ACS) on the Hubble Space Telescope. The sky coverage of M87 is $100 \%$ inside a projected galactocentric radius $R_{p}$ of $6.4 \mathrm{kpc}$, and is $\gtrsim 40 \%$ inside $R_{p}=10 \mathrm{kpc}$ (the imaging radially covers up to $R_{p}=13.9 \mathrm{kpc}$ ).

Jordán et al. (2009) present a table of completeness as a function of magnitude, half-mass radius, and background intensity of the GC. Since the ACS GC catalog provides these values for all GCs found, one can estimate the completeness at the locations of all GCs using this table. We obtained completeness value as a function of radius and magnitude by binning completeness value of individual GCs. The completeness value of a bin was an average of the values of individual GCs in that bin. The completeness becomes worse at smaller $R_{p}$, where the background is brighter. Since we will fit both MF and RD of the GC system, fitting ranges that are wide in both $\mathrm{MF}$ and $\mathrm{RD}$ are desired. We choose fitting ranges of $3 \mathrm{kpc}<R_{p}<10 \mathrm{kpc}$ and $18.6 \mathrm{mag}<M_{z}<24.4 \mathrm{mag}$, which give a minimum completeness of $75 \%$.

We constructed a completeness-corrected luminosity function (LF) for these radial and magnitude ranges, then converted it into an MF using a mass-to-light $(\mathrm{M} / \mathrm{L})$ ratio of 2.67 from Jordán et al. (2007). The mass range of this $\mathrm{MF}$ is $10^{4.67} \mathrm{M}_{\odot}<M<10^{7} \mathrm{M}_{\odot}$. This (completeness-corrected) MF has a total GC number and mass of 1517 and $7.5 \times 10^{8} \mathrm{M}_{\odot}$, respectively. Although the Milky Way GCs are found to have different M/L values for blue and red GCs (see below for discussion on color bimodality), we use the same M/L value for both GC groups of M87 here. This is because 1) there are no reliable $M / L$ estimates for the two GC groups in M87 yet, and 2) different GC systems appear to have different relations between $\mathrm{M} / \mathrm{L}$ and color (Strader et al. 2009), so it is probably not appropriate to apply M/L values of one galaxy (e.g., Milky Way) to M87.

As in most galaxies, the GC system in M87 also has bimodality in its color distribution. In the present paper, we also fit our simulations to blue and red GCs separately. We divide GCs into blue and red ones by their $g-z$ color (blue if $g-z<1.17 \mathrm{mag}$, red otherwise). The total number and total mass $M_{T}$ of blue GCs in the above radial and mass ranges are 607 and $3.2 \times 10^{8} \mathrm{M}_{\odot}$, and those of red GCs are 912 and $4.3 \times 10^{8} \mathrm{M}_{\odot}$.

\section{MODELS AND INITIAL CONDITIONS}

We adopt the anisotropic FP model used in SKT, which is originally developed by Takahashi \& Lee (2000; and references therein). The model integrates the orbitaveraged FP equation of two (energy-angular momentum) dimensions and considers multiple stellar mass components and the effects of three-body and tidalcapture binary heating, stellar evolution, tidal fields, disk/bulge shocks, dynamical friction, and realistic (eccentric and shrinking) cluster orbits (see SKT for the detailed implementation of dynamical friction and realistic orbits). The model implements an Alternating Direction Implicit method developed by Shin \& Kim (2007) for integrating the 2-dimensional FP equation with better numerical stability. This FP model is in good agreement with N-body methods for clusters not only on circular orbits but also on eccentric orbits. A comparison between the FP calculations and N-body simulations of Baumgardt \& Makino (2003) for clusters on eccentric orbits with initial masses larger than $10^{4} \mathrm{M}_{\odot}$ shows a good agreement of cluster lifetimes within $\sim 25 \%$.

Variables of our FP survey are $M$, apocenter of the orbit $R_{\text {apo }}$, and orbit eccentricity $e$. We choose eight $M$ values from $10^{4} \mathrm{M}_{\odot}$ to $10^{7.5} \mathrm{M}_{\odot}$ and six $R_{\text {apo }}$ values from $10^{3} \mathrm{pc}$ to $10^{5.5} \mathrm{pc}$, both equally spaced on the logarithmic scale. For the orbit eccentricity, we choose $e=0,0.25,0.5$, and 0.75 . We perform FP calculations for all possible combinations of these three parameters. The total number of cluster models considered in our study amounts to 192 .

The stellar density and velocity dispersion distributions within each cluster follow the King model (King 1966) with a concentration parameter $W_{0}=7$ and with no initial velocity anisotropy or mass segregation. 

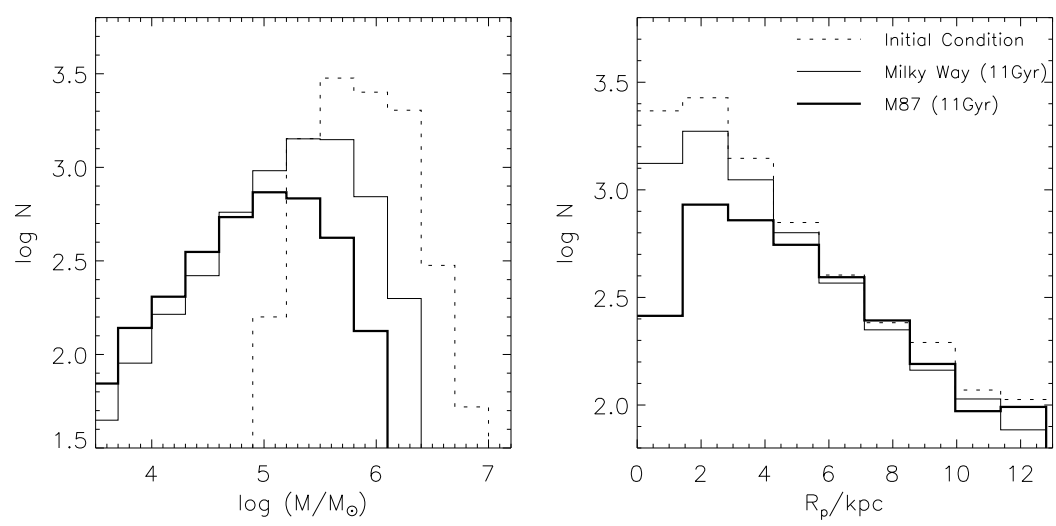

Fig. 2.- MF (left panel) and RD (right panel) at $11 \mathrm{Gyr}$ in two different galactic potentials: the Milky Way potential (thin solid line) and M87 potential (thick solid line). The initial MF and RD are the same for both cases: they are the best-fit initial GC MF and RD obtained for the Milky Way by SKT.

Clusters are assumed to initially fill the tidal radius at the pericenter $R_{\text {peri }}$ following Baumgardt (1998), and initially located at $R_{\text {apo }}$. For the initial stellar mass function within each cluster, we adopt the model by Kroupa (2001) with a mass range of $0.08-15 \mathrm{M}_{\odot}$, which is realized by 15 discrete mass components in our FP model.

Parmentier \& Gilmore (2007) find that a power-law initial mass distribution of protoglobular clouds can quickly evolve into a log-normal initial GCMF due to the expulsion of the gas remnant from star formation if the power-law mass distribution has a lower mass limit. Our initial GCMFs are to be regarded as the models after the gas expulsion.

We adopt the same stellar and velocity dispersion distributions for both blue and red GC groups because nothing is known yet about the dependence of these distributions on GC color. We also assume that the initial orbit eccentricity distribution is the same for both blue and red GC groups. Although the GCs in M87 do show figure rotation, the amplitudes of the rotations for the blue and red GC groups are not too different (Cote et al. 2001). Thus the observed rotation of GCs is probably due to the rotation of the mother galaxy, which does not significantly affect the assumptions and results in the present paper.

\section{BEST-FIT INITIAL MASS FUNCTION AND RADIAL DISTRIBUTION OF M87 GLOBULAR CLUSTER SYSTEM}

\subsection{Synthesis of Fokker-Planck Calculations}

As discussed in Section 3, we performed a total of 192 FP calculations with different initial cluster conditions in 3-dimensional parameter space, $M, R$, and $e$. The goal of our study is to find the best-fit initial distributions of these variables, and for this, we adopt a log-normal function,

$$
\mathrm{d} N(M) \propto \exp \left\{-0.5\left[\log \left(M / M_{p}\right) / \sigma_{\log M}\right]^{2}\right\} \mathrm{d} M,
$$

and a Schechter function,

$$
\mathrm{d} N(M) \propto M^{\alpha} \exp \left(-M / M_{s}\right) \mathrm{d} M,
$$

for initial MFs, and a softened power-law function,

$$
\mathrm{d} N(R) \propto 4 \pi R^{2} \mathrm{~d} R /\left[1+\left(R / R_{0}\right)^{\beta}\right],
$$

for initial RDs. We assume that the initial MF is independent of $R$. For the sake of simplicity, we do not parameterize the $e$ distribution, and adopt a fixed isotropic distribution, $\mathrm{d} N(e)=2 e \mathrm{~d} e$.

We evolve all of our FP models for 11 Gyrs (SKT evolved their models for 13 Gyrs), considering that M87 probably underwent a series of major mergers during the first 2-3 Gyrs of its lifetime (thus our initial MF and $\mathrm{RD}$ would be in fact those right after the major merger period). This approach has a problem that stellar evolution in GCs starts at 2-3 Gyrs. This will somewhat delay the evolution of stellar mass function and mass segregation in GCs, but the effect of the dealy is relatively less important to the overall evolution of the GC system compared to other processes such as dynamical friction and mass loss over tidal radius. We assume that the GC system of M87 has not been significantly disturbed by later merger events even though such dichotomy is an oversimplification.

After all 192 FP calculations are done, the aforementioned set of initial MF and RD models are used to search for the best-fit initial GC distributions in 3dimensional parameter space: $\left(M_{p}, \sigma_{\log M}, \beta\right)$ for the log-normal MF and $\left(\alpha, M_{s}, \beta\right)$ for the Schechter MF. We synthesize our 192 FP calculations with appropriate weights to produce a given initial $\mathrm{MF}$ and $\mathrm{RD}$, and find a set of $\left(M_{p}, \sigma_{\log M}, \beta\right)$ or $\left(\alpha, M_{s}, \beta\right)$ that best fits the present-day MF and RD of the M87 GC system. 
Table 1 .

Best-fit parameters for initial GC distributions

\begin{tabular}{|c|c|c|c|c|c|c|c|c|c|c|}
\hline \multirow[b]{2}{*}{ GC Group } & \multirow[b]{2}{*}{ MF } & \multicolumn{2}{|c|}{ Log-normal MF } & \multicolumn{2}{|c|}{ Schechter MF } & \multirow[b]{2}{*}{$\beta$} & \multicolumn{2}{|c|}{$M_{T}$} & \multirow[b]{2}{*}{$\chi^{2}$} & \multirow[b]{2}{*}{$p$-value } \\
\hline & & $\log M_{p}$ & $\sigma_{\log M}$ & $\log M_{s}$ & $\alpha$ & & 0 Gyr & $11 \mathrm{Gyr}$ & & \\
\hline All GCs & $\mathrm{L}$ & $5.20_{-.10}^{+.10}$ & $0.52_{-.02}^{+.02}$ & & & $2.93_{-.09}^{+.09}$ & $1.8 \times 10^{10}$ & $6.1 \times 10^{9}$ & 10.0 & $44 \%$ \\
\hline All GCs & $\mathrm{S}$ & & & $6.53_{-.02}^{+.05}$ & $1.96_{-.05}^{+.08}$ & $2.97_{-.10}^{+.12}$ & $1.7 \times 10^{10}$ & $5.6 \times 10^{9}$ & 21.0 & $2 \%$ \\
\hline Blue GCs & $\mathrm{L}$ & $5.42_{-10}^{+.07}$ & $0.47_{-02}^{+.02}$ & & & $2.66_{-14}^{+.14}$ & $1.1 \times 10^{10}$ & $4.0 \times 10^{9}$ & 10.1 & $43 \%$ \\
\hline Blue GCs & $\mathrm{S}$ & & & $6.52_{-.05}^{+.05}$ & $1.82_{-.10}^{+.10}$ & $2.69_{-.14}^{+.14}$ & $1.1 \times 10^{10}$ & $3.9 \times 10^{9}$ & 10.7 & $39 \%$ \\
\hline Red GCs & $\mathrm{L}$ & $5.11_{-.17}^{+.15}$ & $0.53_{-.04}^{+.04}$ & & & $3.13_{-.10}^{+10}$ & $8.2 \times 10^{9}$ & $2.5 \times 10^{9}$ & 13.8 & $18 \%$ \\
\hline Red GCs & S & & & $6.52_{-.09}^{+.09}$ & $2.03_{-.11}^{+.11}$ & $3.17_{-.10}^{+.10}$ & $7.8 \times 10^{9}$ & $2.3 \times 10^{9}$ & 20.5 & $2 \%$ \\
\hline MW GCs & $\mathrm{L}$ & $5.61_{-15}^{+.11}$ & $0.33_{-05}^{+.06}$ & & & $4.24_{-21}^{+.21}$ & $1.7 \times 10^{8}$ & $4.5 \times 10^{7}$ & 12.9 & $88 \%$ \\
\hline
\end{tabular}

Note.-Best-fit initial distribution parameters for three different GC groups and two different MF forms. "L" in the MF column stands for the log-normal function and "S" for the Schechter function. The $p$-value is the probability of having a $\chi^{2}$ that is larger than the value obtained from our $\chi^{2}$ test between the model and the observation, whose degree of freedom is 12. The masses are in units of $\mathrm{M}_{\odot}$ and the radii in units of kpc. Best-fit parameters for the Milky Way GC system obtained by Shin et al. (2008) are given at the last row for a comparison. Both initial (0 Gyr) and current (11 Gyr) $M_{T}$ values are from our best-fit models, so they include all radial and mass ranges, wherease the observed $M_{T}$ values given in Section 2 are limited to our fitting ranges, $3 \mathrm{kpc}<R_{p}<10 \mathrm{kpc}$ and $10^{4.67} \mathrm{M}_{\odot}<M<10^{7} \mathrm{M}_{\odot}$.

Unlike in SKT, we fix $R_{0}$ to be $5 \mathrm{kpc}$, instead of using it as a free parameter to be fitted. This is because our $\mathrm{RD}$ fitting range $(3-10 \mathrm{kpc})$ is so small that too large parameter space in $R_{0}-\beta$ plane gives satisfactory fits, making a search for the best-fit parameter set in 4dimensional parameter space not so meaningful. We choose $R_{0}=5 \mathrm{kpc}$ following McLaughlin (1999) who modeled the stellar halo density profile of M87 with a Dehnen profile with a scale length of $5.1 \mathrm{kpc}$.

When finding the best-fit initial MF and RD, we minimize the sum of the $\chi^{2}$ values from both $M$ and $R_{p}$ histograms, which are constructed by using 7 logarithmic bins between $10^{4.67} \mathrm{M}_{\odot}$ and $10^{7} \mathrm{M}_{\odot}$ for $M$ and 7 linear bins between $3 \mathrm{kpc}$ and $10 \mathrm{kpc}$ for $R_{p}$. Since the model $M$ and $R_{p}$ histograms are both constrained by the observed number of clusters in our fitting ranges, the degree of freedom for our $\chi^{2}$ test is 12 . Minimizing the sum of the two $\chi^{2}$ values from MF and $\mathrm{RD}$ cannot consider any correlation between MF and $\mathrm{RD}$, so we will check if the best-fit 11 Gyr model MFs have the $R_{p}$ dependence that is consistent with the observed MF. This way, we would be able to find a model that properly reproduces the observed 2-dimensional ( $M$ and $R_{p}$ ) distribution of GCs.

Unlike $M$, an $R$ value of each FP model evolves with time oscillating between $R_{\text {peri }}$ and $R_{\text {apo }}$, and thus the model RD at $11 \mathrm{Gyr}$ constructed from our 'population synthesis' may suffer a significant random noise. To decrease this noise, we build the model RD by adding up the probability distributions between $R_{\text {peri }}$ and $R_{\text {apo }}$ that are given by the orbital information at $11 \mathrm{Gyr}$.

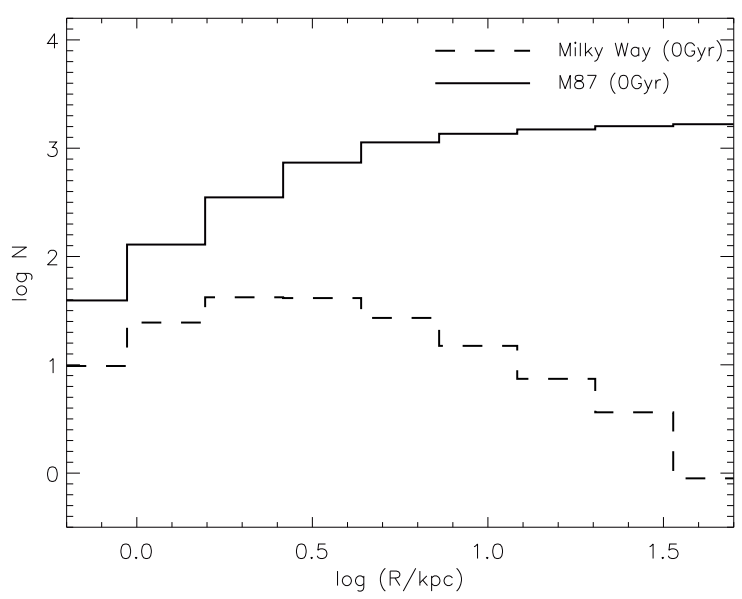

Fig. 3.- Comparison of the best-fit initial radial distribution between the Milky Way and M87 GC systems. Both models are for the log-normal initial GCMF case, and the M87 model is from the present study, while the Milky Way model is from SKT. The initial radial distribution of the M87 GC system is significantly more extended.

\subsection{Evolution of the Milky Way Globular Clus- ter System in M87 Potential}

Before presenting our best-fit initial GC distributions, we first compare the evolution of the same initial GC MF and RD in two different gravitational potentials, those of the Milky Way and M87. For this experiment, we adopt the best-fit GC MF and RD found for the Milky Way by obtained SKT, which is 
$\log M_{p}=4.0 \times 10^{5} \mathrm{M}_{\odot}, \sigma_{\log M}=0.33, \beta=4.24$, and $R_{0}=2.9 \mathrm{kpc}$. Fig. 2 compares the evolved MFs and RDs of this initial GC system in the two potentials, and shows that the GC system in M87 potential loses more mass as expected. The faster evolution of GCs in M87 potential is more prominent for GCs with a large $M$ and/or a small $R_{p}$, as these GCs suffer more effective dynamical friction or mass loss over tidal radius. The fraction of the remaining mass at $11 \mathrm{Gyr}$ is $26 \%$ for the Milky Way and $7 \%$ for M87.

\subsection{Best-fit Initial Globular Cluster Distribu- tions in M87}

We have fit our FP models to three groups of observed GCs: all GCs, blue GCs only, and red GCs only. The best-fit parameters obtained from our $\chi^{2}$ tests are presented in Table 1 along with those obtained for the Milky Way GC system by SKT (the log-normal initial GCMF case only, because SKT did not try a Schechter initial GCMF).

Let us first discuss our fits for all GCs in M87. The $p$-value (significance level of a statistical test) from our $\chi^{2}$ test is acceptably high for the log-normal initial GCMF (44\%), but is a bit low for the Schechter initial GCMF (2.1\%), implying that a log-normal function better describes the initial MF and RD of the M87 GC system (see Figs. 4 and 5). In case of a log-normal initial GCMF, M87 has about 0.4 dex lower $\log M_{p}$, but $\sim 0.2$ dex higher $\log M$ than those found for the Milky Way by SKT. M87's having a lower $\log M_{p}$ than the Milky Way may be a bit of surprise, considering that M87 is a much bigger galaxy. This result could be understood as a result of complex merger history in the early phase of M87. The galaxy is found to have a much shallower initial RD than the Milky Way, and this can be also interpreted as a result of merger events during the first 2-3 Gyr of the galaxy as mixing of GCs during a merging will lessen the central concentration of GCs. The simulations performed by Bekki \& Forbes (2006) indeed show that the radial density profile of GC system becomes flatter after a series of merger events.

The initial and current $M_{T}$ 's in M87 GCs are found to be $1.8_{-0.2}^{+0.3} \times 10^{10} \mathrm{M}_{\odot}$ and $6.1_{-0.8}^{+0.9} \times 10^{10} \mathrm{M}_{\odot}$ for the log-normal initial GCMF case. This initial $M_{T}$ is about 100 times bigger than that of the Milky Way GC system. One may expect M87 to have a much smaller current-to-initial $M_{T}$ ratio due to its significantly larger $M_{e n c}$ profile (see Section 4.2), but we find that M87 has a slightly larger current-to-initial $M_{T}$ ratio than the Milky Way (34\% vs. 26\%). This is because the M87 GC system has a considerably larger initial radial distribution (larger $R_{0}$ and smaller $\beta$; see Fig. 3), which causes GCs to have larger tidal radii (slower evaporation) and to suffer less dynamical friction. The initial $M_{T}$ and the remaining mass fraction at $11 \mathrm{Gyr}$ found for the Schechter initial GCMF are similar to those for the log-normal case.

GC systems of most galaxies, including M87, show bimodality in color distribution, and this is generally interpreted as a bimodality in metallicity distribution. ${ }^{\ddagger}$ It is natural to conceive that these two different GC groups have different origins, thus here we fit our FP models to blue and red GC groups separately and see if their initial MFs and RDs are significantly different in a statistical sense (see Figs. 6 through 9).

When only blue GCs are fitted, the $p$-values from $\chi^{2}$ tests are acceptably high for both log-normal and Schechter initial GCMFs, but the $p$-value for the red GCs is marginally acceptable for the log-normal initial GCMF and unacceptably low for the Schechter initial GCMF. In case of the log-normal initial GCMF, blue GCs have greater $\log M_{p}$ and smaller $\sigma_{\log M}$ than all GCs, while red GCs have slightly smaller $\log M_{p}$ and slightly greater $\sigma_{\log M}$ than all GCs. The significances of differences between the two groups are 1.8- $\sigma$ for $\log M_{p}, 1.3-\sigma$ for $\sigma_{\log M}$, and 2.7- $\sigma$ for $\alpha$. These statistical values imply that the two GC groups appear to have started from different initial MFs and RDs.

The initial $M_{T}$ in blue GCs is found to be somewhat larger than that in red GCs for both log-normal and Schechter initial GCMFs. Although the observed total mass of red GCs are larger than blue GCs (see Section 2), the current total mass estimated from our fit is larger for blue GCs. This is because blue GCs currently have flatter RD than red GCs, so there is a larger difference between the observed (covering $3 \mathrm{kpc}<$ $R_{p}<10 \mathrm{kpc}$ ) and estimated (covering all radial ranges) total masses for the blue GCs.

To see if our best-fit models agree with the observed $R_{p}$ dependence of the MF, we calculate $\chi^{2}$ for the differences of $\langle\log M\rangle$ and $\sigma_{\log M}$ between the model and the observation:

$$
\begin{aligned}
\chi^{2}(\langle\log M\rangle) & =\sum_{j} \frac{\left(\left\langle\log M_{o, j}\right\rangle-\left\langle\log M_{m, j}\right\rangle\right)^{2}}{\sigma_{\log M_{m, j}} / N_{o, j}} \\
\chi^{2}\left(\sigma_{\log M}\right) & =\sum_{j} \frac{\left(\sigma_{\log M_{o, j}}-\sigma_{\log M_{m, j}}\right)^{2}}{\sigma_{\log M_{m, j}}^{2} / 2 N_{o, j}}
\end{aligned}
$$

where subscripts $o$ and $m$ stand for the observation and the model, respectively, and subscript $j$ represents the radial bin (we use five radial bins for this test; see right panels of Figs. 4 through 9). We find that the $p$-values from the above $\chi^{2}$ tests for our best-fit MFs are about $20 \%$ for all GCs, about $60 \%$ for blue GCs, and about $40 \%$ for red GCs. Thus the $R_{p}$ dependence of $\log M$ and $\sigma_{\log M}$ from our best-fit MF is slightly better when red and blue GC groups are fit separately, but the $p$ values for all GCs are still not unacceptably low.

\footnotetext{
$\ddagger$ Note that Yoon et al. (2006) argue that the color bimodalities in some galaxies can be explained even without metallicity bimodalities if the relationship between color and metallicity is assumed to be nonlinear.
} 

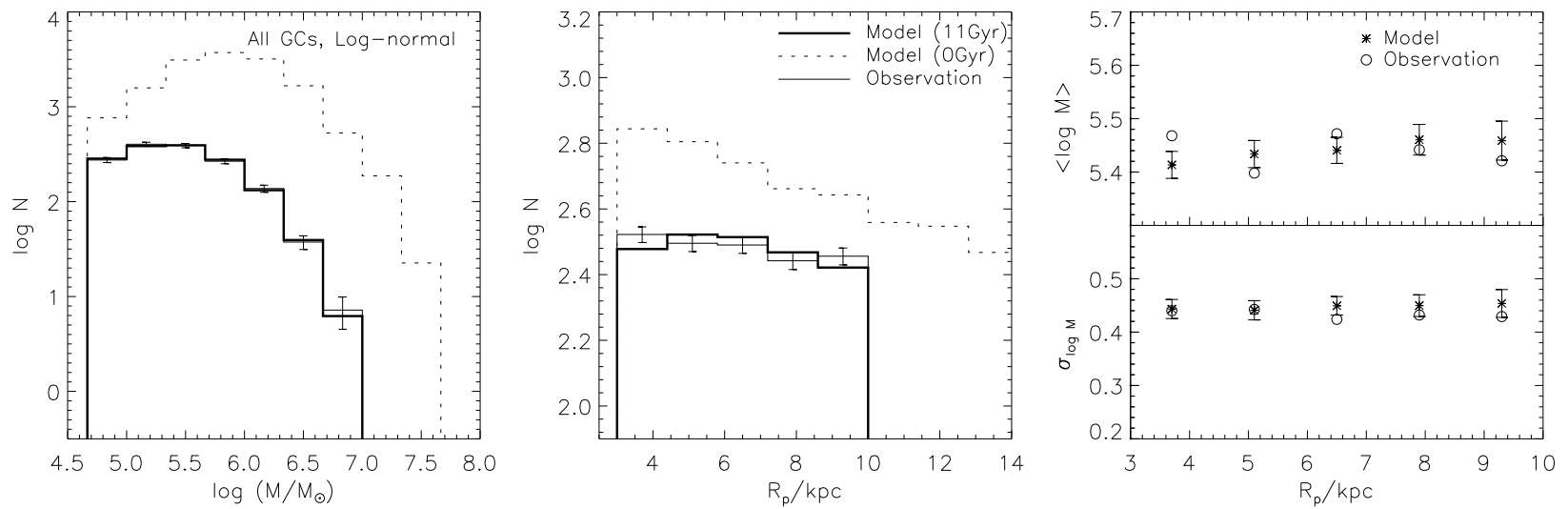

Fig. 4.- $M$ (left panel) and $R_{p}$ (middle panel) histograms of our best-fit models at 11 Gyr (thick solid lines) for a lognormal initial GCMF case and for all GCs (thin solid lines). Also shown are the initial $M$ and $R_{p}$ histograms of our best-fit models (dotted lines) and those from the observations (thin solid lines). The right panel shows $\langle\log M\rangle$ and $\sigma_{\log M}$ values of the best-fit log-normal model (asterisks) and the observation (open circles) for five radial bins. Error bars indicate the 1- $\sigma$ uncertainties for the model.
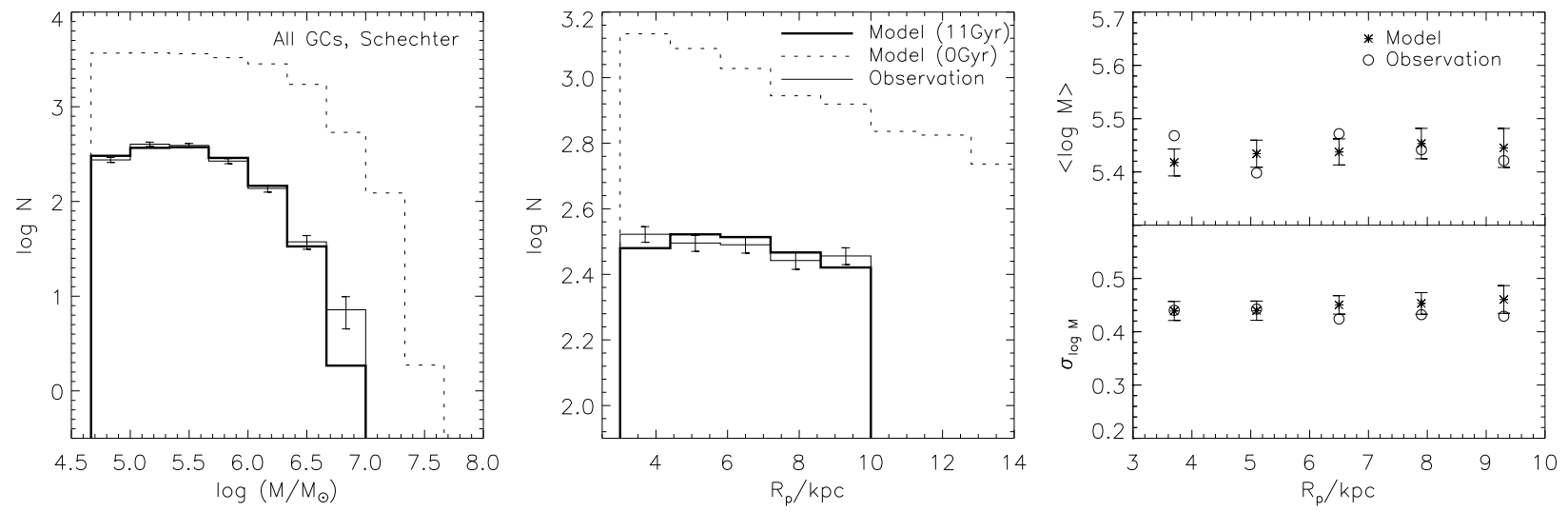

Fig. 5.- Same as Fig. 4, but for the Schechter initial GCMF.

\section{SUMMARY AND DISCUSSION}

We have calculated the dynamical evolution of the M87 GC system using the most advanced and realistic FP model. By comparing our calculations with both $\mathrm{MF}$ and RD of the observed GC system, we found the best-fit initial distributions for three GC groups: all GCs, blue GCs, and red GCs. The log-normal initial GCMFs generally give better fits to the observations than the Schechter initial GCMFs. When compared to the GC system of the Milky Way, that of M87 initially has a smaller $\log M_{p}$ and a larger $\sigma_{\log M}$. The initial total mass in GCs is estimated to be $1.8_{-0.2}^{+0.3} \times 10^{10} \mathrm{M}_{\odot}$, which is about 100 times larger than that of the Milky Way. However, in spite of the much deeper potential of M87, the two GC systems have similar fractions of remaining masses in GCs (34\% for M87 and $26 \%$ for the Milky Way). This is because the M87 GC system is more extensively distributed in the beginning than the Milky Way GC system. When the blue and red GCs are fitted separately for the log-normal initial GCMF, the blue GCs are found to initially have a $\operatorname{larger} \log M_{p}$, a larger $M_{T}$, and a smaller $\beta$ (shallower RD) than the red GCs. The best-fit initial MFs and RDs of blue and red GC groups are different from each other at 2 to $3-\sigma$ levels.

There are three leading scenarios for metallicity (color) bimodality. 1) The major merger model (Ashman \& Zepf 1992): the metal-poor GCs are from the progenitor spirals and the metal-rich GCs are formed in the gas-rich merger. 2) The "in situ" model (Forbes, Brodie \& Grillmair 1997): the metal-poor GCs were formed during the earliest phases of galaxy formation, and the metal-rich GCs and the bulk of the galaxy field stars were formed in the second phase. 3) The accretion model (e.g., Côté, Marzke \& West 1998): the metalrich GCs were formed in a massive seed galaxy, and the metal-poor GCs were acquired in the dissipation- 

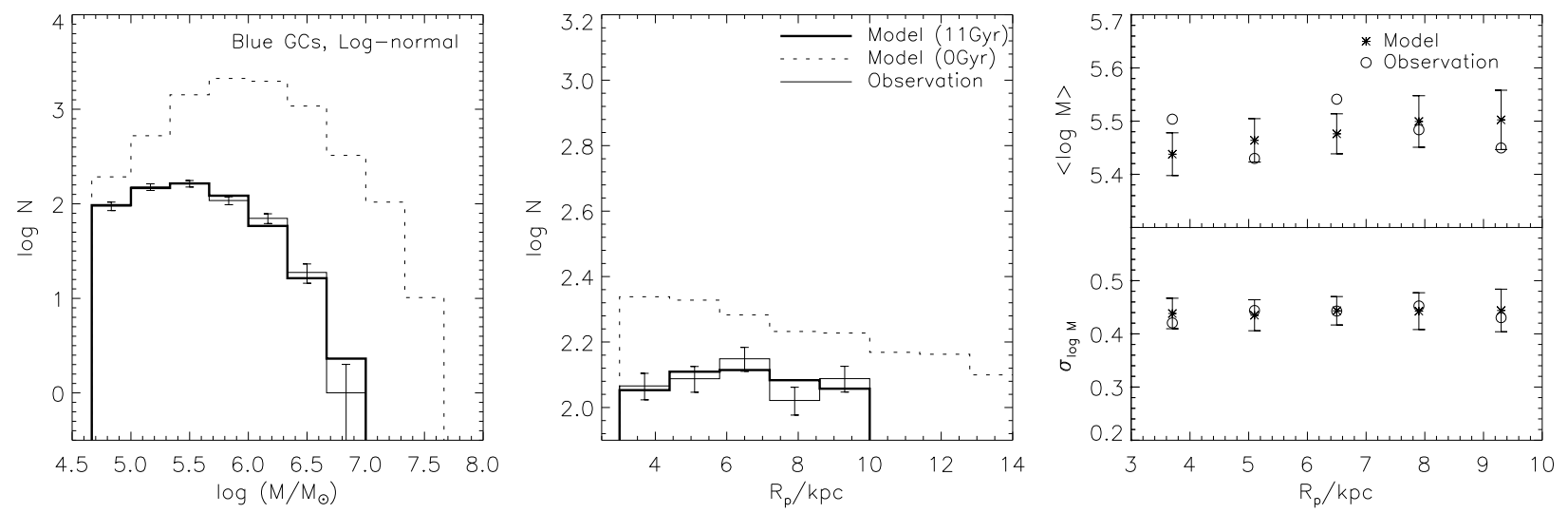

Fig. 6. - Same as Fig. 4, but for the blue GCs only.
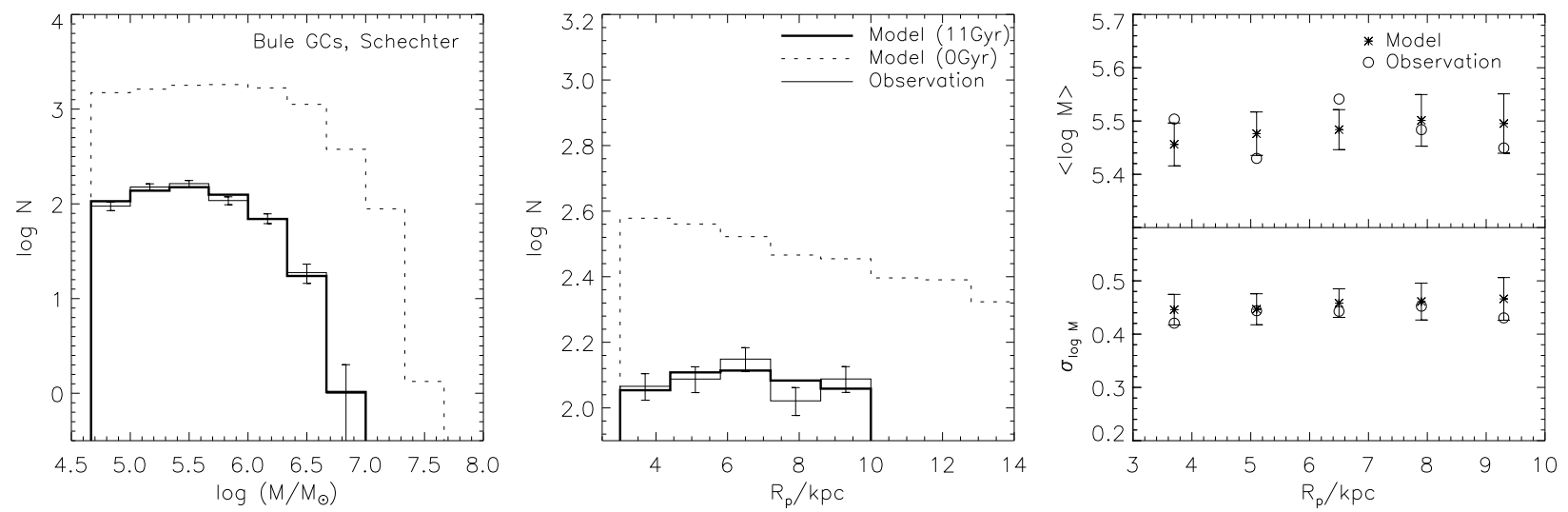

Fig. 7. - Same as Fig. 5, but for the blue GCs only.

less accretion of nearby lower-mass galaxies.

Among these three scenarios, our results support the major merger model most. Griffen et al. (2009) analyzed the Aquarius simulation (Springel et al. 2008), the highest resolution simulation of Milky Way sized halos so far, and found that the initial distribution of the metal-rich (red) GCs in the simulation is centrally concentrated as in our results. Furthermore, Bekki \& forbes (2006) found that the radial density profiles of the existing (metal-poor or blue) GC systems in elliptical galaxies become progressively flatter as the galaxies undergo more merger events. Our initial GC MF and $\mathrm{RD}$ distributions are targeted for the epoch when the major merging events has ended, thus our finding that the blue (metal-poor) GCs initially had flatter RD than the red GCs is consistent with the major merger scenario for metallicity bimodality observed in GC systems including M87.

\section{ACKNOWLEDGMENTS}

We thank Holger Baumgardt, Narae Hwang, Ho Seong Hwang, Myung Gyoon Lee, and Suk Jin Yoon for helpful discussion, and the anonymous referee for valuable comments. This work was supported by a grant from Kyung Hee University in 2008 (KHU-20080650).

\section{REFERENCES}

Baumgardt, H. 1998, The Initial Distribution and Evolution of Globular Cluster Systems, A\&A, 330, 480

Baumgardt, H., \& Makino, J. 2003, Dynamical Evolution of Star Clusters in Tidal Fields, MNRAS, 340, 227

Bekki, K., \& Forbes, D. A. 2006, On the Structure of Globular Cluster Systems in Elliptical Galaxies, A\&A, 445, 485

Côté, P., McLaughlin, D. E., Hanes, D. A., Bridges, T. J., Geisler, D. Merritt, D., Hesser, J., Harris, G. L. H., \& Lee, M. G. 2001, Dynamics of the Globular Cluster System Associated with M87 (NGC 4486). II. Analysis, ApJ, 559, 828

Côté, P., Blakeslee, J. P., Ferrarese, L., Jordán, A., Mei, S., Merritt, D., Milosavljević, M., Peng, E. W., 

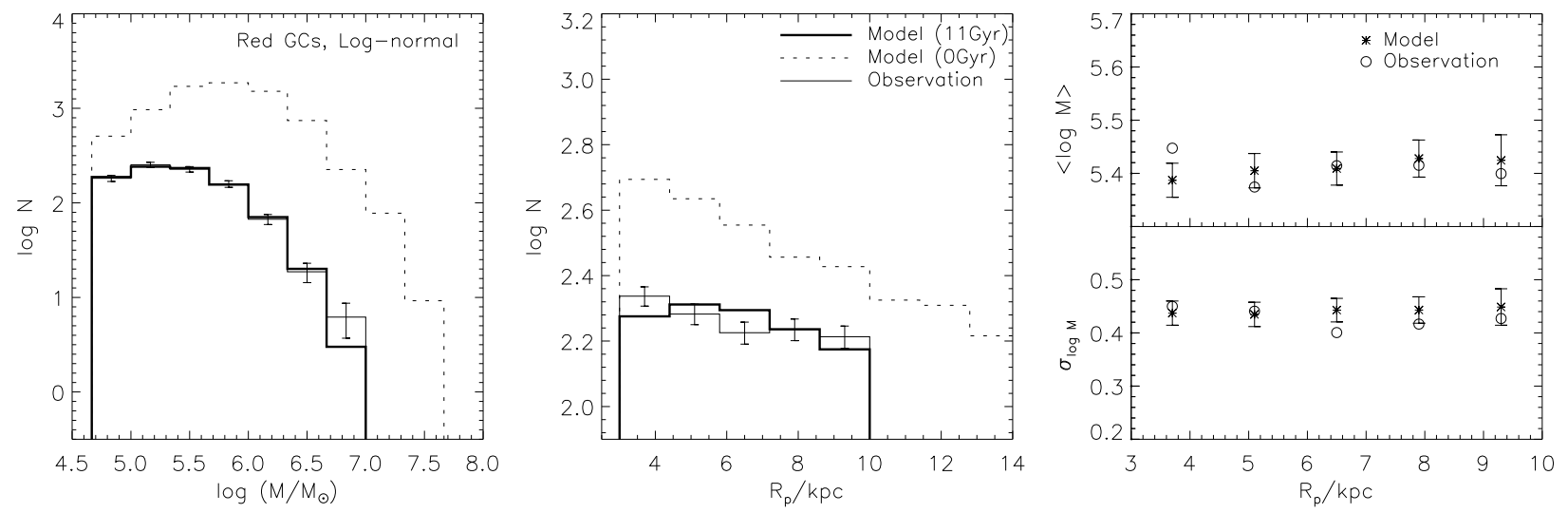

Fig. 8.- Same as Fig. 4, but for the red GCs only.
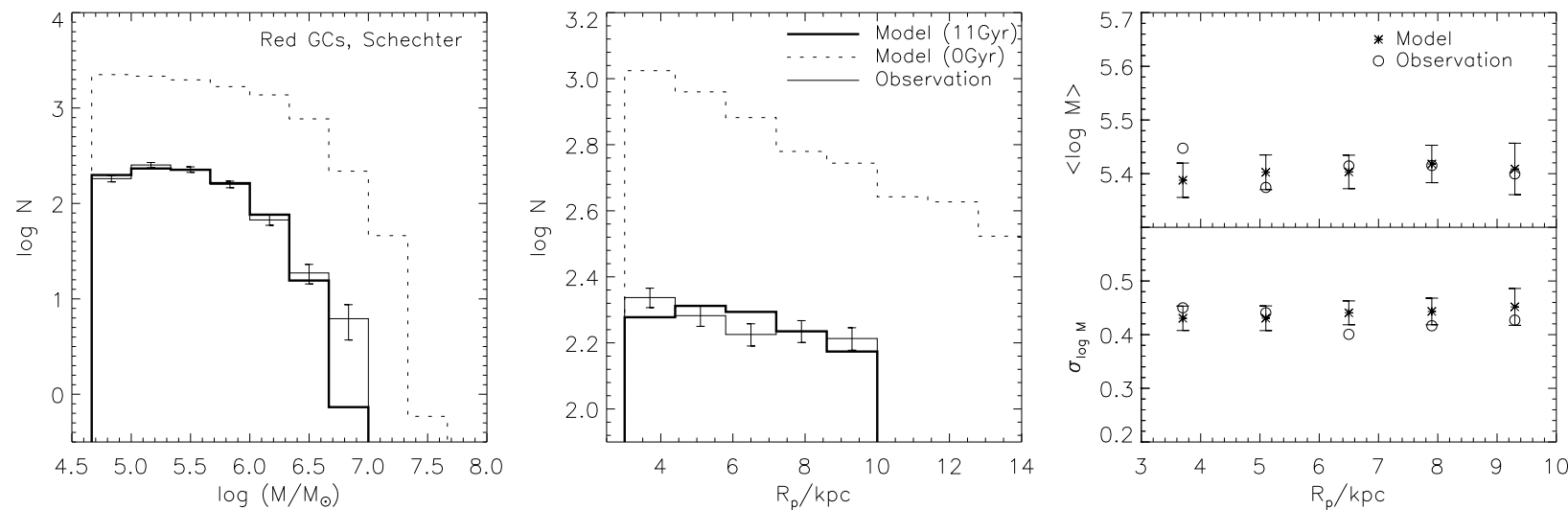

Fig. 9.- Same as Fig. 5, but for the red GCs only.

Tonry, J. L., \& West, M. J. 2004, The ACS Virgo Cluster Survey. I. Introduction to the Survey, ApJS, 153,223

Johnston, K. V., Spergel, D. N., \& Hernquist, L. 1995, The Disruption of the Sagittarius Dwarf Galaxy, ApJ, 451, 598

Jordán, A., McLaughlin, D. E., Côté, P., Ferrarese, L., Peng, E. W., Mei, S., Villegas, D., Merritt, D., Tonry, J. L., \& West, M. J. 2007, The ACS Virgo Cluster Survey. XII. The Luminosity Function of Globular Clusters in Early-Type Galaxies, ApJS, 171,101

Jordán, A., Peng, E. W., Blakeslee, J. P., Côté, P., Eyheramendy, S., Ferrarese, L., Mei, S., Tonry, J. L., \& West, M. J. 2009, The ACS Virgo Cluster Survey XVI. Selection Procedure and Catalogs of Globular Cluster Candidates, ApJS, 180, 54

McLaughlin, D. E. 1999, Evidence in Virgo for the Universal Dark Matter Halo, ApJ, 512, L9

King, I. R. 1966, The Structure of Star Clusters. III. Some Simple Dynamical Models, AJ, 71, 64
Kroupa, P. 2001, On the Variation of the Initial Mass Function, MNRAS, 322, 231

Parmentier, G., \& Gilmore, G. 2007, The Origin of the Gaussian Initial Mass Function of Old Globular Cluster Systems, MNRAS, 377, 352

Peng, E. W., Jordán, A., Côté, P., Takamiya, M., West, M. J., Blakeslee, J. P., Chen, C. W., Ferrarese, L., Mei, S., Tonry, J. L., \& West, A. A. 2008, The ACS Virgo Cluster Survey. XV. The Formation Efficiencies of Globular Clusters in Early-Type Galaxies: The Effects of Mass and Environment, ApJ, 681, 197

Schechter, P. 1976, An Analytic Expression for the Luminosity Function for Galaxies, ApJ, 203, 297

Shin, J., \& Kim, S. S. 2007, Alternating Direction Implicit Method for Two-Dimensional Fokker-Planck Equation of Dense Spherical Stellar Systems, JKAS, 40,91

Shin, J., Kim, S. S., \& Takahashi, K. 2008, Dynamical Evolution of the Mass Function and Radial Profile of the Galactic Globular Cluster System, MNRAS, 386, L67 (SKT) 
Strader, J., Smith, G. H., Larsen, S., Brodie, J. P., Huchra, J. P. 2009, Mass-to-Light Ratios for M31 Globular Clusters: Age Dating and a Surprising Metallicity Trend, AJ, 138, 547

Takahashi, K., \& Lee, H. M. 2000, Evolution of Multimass Globular Clusters in the Galactic Tidal Field with the Effects of Velocity Anisotropy, MNRAS, 316,671

Yoon, S. J., Yi, S. K, \& Lee, Y. W. 2006, Explaining the Color Distributions of Globular Cluster Systems in Elliptical Galaxies, Science, 311, 1129

Zhang, Q., \& Fall, M. 1999, The Mass Function of Young Star Clusters in the "Antennae" Galaxies, ApJ, 527, 81 